\title{
A 80Ms/sec 10bit PIPELINED ADC Using 1.5Bit Stages And Built-in Digital Error Correction Logic
}

\author{
P.Prasad Rao ${ }^{1}$ and Prof.K.Lal Kishore ${ }^{2}$, \\ ${ }^{1}$ Research Scholar, JNTU-Hyderabad \\ prasadrao_hodeyahoo.co.in \\ ${ }^{2}$ Director, R\&D, JNTU-Hyderabad \\ lalkishorek@yahoo. com
}

\begin{abstract}
Use of pipelined ADCs is becoming increasingly popular both as stand alone parts and as embedded functional units in SOC design. They have acceptable resolution and high speed of operation and can be placed in relatively small area. The design is implemented in $0.18 u M$ CMOS process. The design includes a folded cascode op-amp with a unity gain frequency of $200 \mathrm{MHz}$ at 88 deg. Phase margin and a dc gain of $75 \mathrm{~dB}$. The circuit employs a built in sample and hold circuit and a three phase non-overlapping clock.
\end{abstract}

\section{Keywords}

ADC, 1.5 bit stage, CMFB, Pipeline, Redundancy bit removal algorithm

\section{INTRODUCTION}

High resolution, high speed CMOS ADCs are used for scanners, high definition TVs, medical equipment, camcorders and radar systems and are limited to 10 bits because of various reasons. In this work, a fully differential 10 bit ADC was implemented using an inherent digital error correction technique. The fully differential Folded Cascode op-amp architecture is used. Power dissipation in op-amp can be reduced by reducing either supply voltage or total current in the circuit or by reducing the both[10]. As the input current is lowered though power dissipation is reduced, the dynamic range is degraded. As the supply voltage decreases, it also becomes increasingly difficult to keep transistors in saturation with the voltage headroom available. The design takes care of power optimization at every level of design. The block diagram of general Pipelined ADC is shown in Fig. (1). Each stage contains a S/H circuit, a low resolution A/D subconverter, a low resolution D/A converter and a differencing fixed gain amplifier. Flash and subranging architectures need exponential rather than linear increase in area to increase their resolution and also require trimming and calibration. But for pipelined converters, area is small and is linearly related to the resolution because the resolution can be increased by adding stages to the end of the pipeline without increasing the number of clock phases required per conversion.

\section{OP-AMP REQUIREMENTS FOR ADCS}

The typical gain and phase responses of an op-amp are shown in fig.(2). The bandwidth and gain characteristics are crucial in the design of data converters. The op-amp is preferred to have $90^{\circ}$ 
International Journal of VLSI design \& Communication Systems (VLSICS) Vol.2, No.3, September 2011 phase margin over full load conditions and process variations to avoid second order step response and its associated ringing.

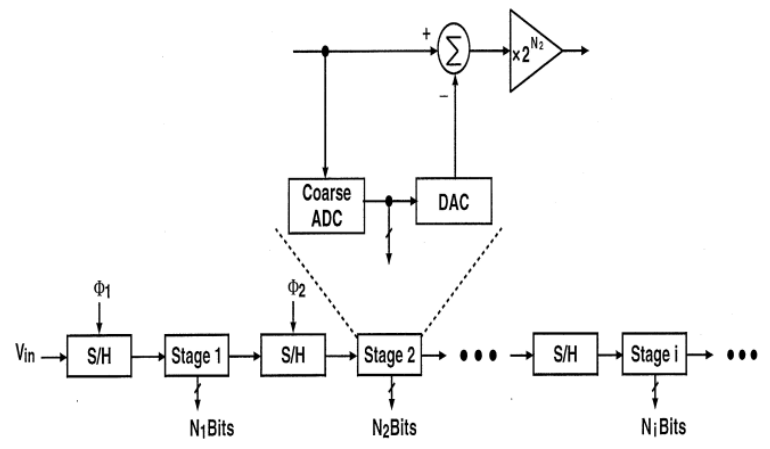

Figure 1. Block diagram of pipelined ADC

Decreasing the phase margin results in an increase of amplitude of ringing and this can increase the settling time. It can be proved that the DC open loop gain of an op-amp used in an ADC must satisfy the condition[1]

$$
\mathrm{A}_{\mathrm{ol}}>=2^{\mathrm{N}+2}
$$

Where $\mathrm{N}$ is the no. of bits of conversion. For example a 10 Bit data converter needs a minimum DC open loop gain of $2^{12}$. The speed of an op-amp is decided by the op-amp used. The minimum unity gain frequency $\left(f_{u}\right)$ for a given settling time $\left(t_{s}<1 / f_{c l k}\right)$ required to settle the output to within $\neq 1 / 2$ LSB of its final value can be evaluated as[1]

$$
\mathrm{f}_{\mathrm{u}}>=0.22(\mathrm{~N}+1) \mathrm{f}_{\mathrm{clk}_{-}}
$$

i.e. for a 10 bit $\mathrm{ADC}$ at $80 \mathrm{MHz}$ clock frequency needs an op-amp with a unity gain frequency of around $200 \mathrm{MHz}$.

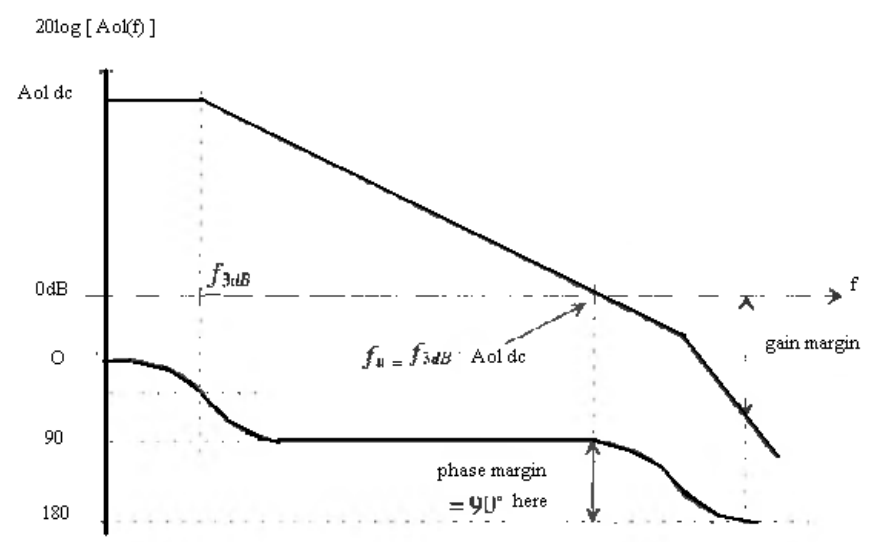

Figure 2. Magnitude and phase response of an op-amp 


\subsection{Folded Cascode Op-Amp}

Modern integrated CMOS op-amps are designed to drive capacitive loads[7][8]. With only a capacitive load it is not necessary to use a buffer at the output for a low impedance node. Therefore, it is possible to design op-amps at higher speeds and larger voltage swings than those which drives resistive loads. These improvements are achieved with a single high impedance node at the output that drives only capacitive loads. For folded cascode op-amps the compensation is achieved by $C_{L}$ itself and it is dominant pole compensation. As $C_{L}$ increases, the op-amp stability improves but is slowed down. The folded cascode op-amp schematic is shown in Fig. ( 3 ).

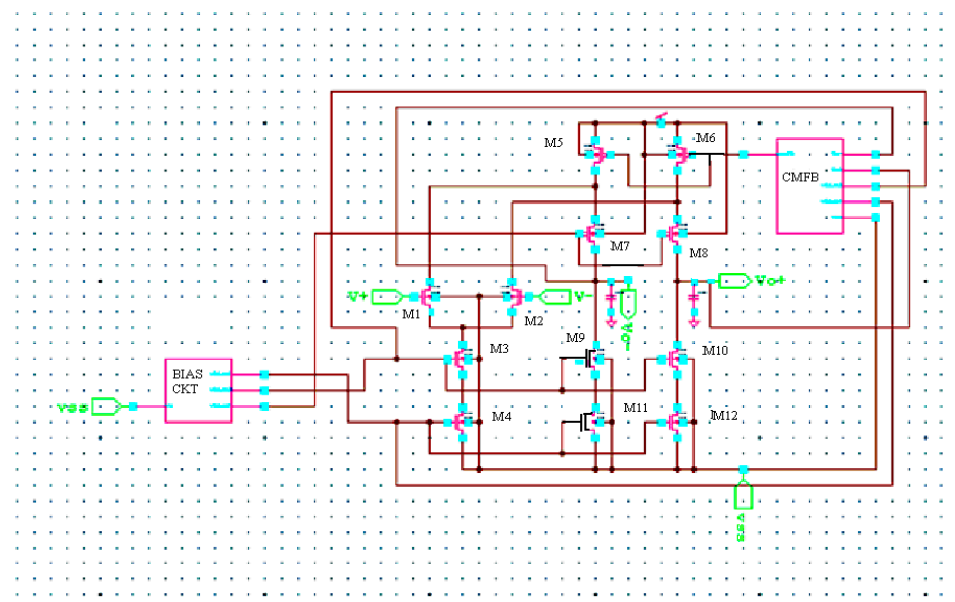

Figure 3. Fully differential folded cascode op-amp

The basic idea of folded cascode op-amp is to apply the opposite type PMOS cascode transistors to the input differential pair of NMOS type. This arrangement allows the output to be taken at the same bias levels as that of input signal. Even though it is a single stage, the gain is reasonable since the gain is decided by the product of input transconductance and the large output impedance. Applying AC input voltage $v_{\text {in }}$ causes the drain of differential stage to be $g_{m} v_{i n}$. This AC drain current is mirrored in the cascaded MOSFETs M7-M12.the output voltage of folded cascode is then

$$
\begin{gathered}
\mathrm{V}_{\text {out }}=\mathrm{g}_{\mathrm{m}} \mathrm{V}_{\text {in }} \mathrm{R}_{\text {out }} \text {. Where } \\
\mathrm{R}_{\text {out }}=\underset{ }{[(\mathrm{R} \text { looking back into the drain of M10 }) \mathrm{II}} \\
\quad(\mathrm{R} \text { looking into the drain of M8 })] . \\
=\left[\mathrm{r}_{\text {out10 }}\left(1+\mathrm{g}_{\mathrm{m} 10} \mathrm{r}_{\text {out12 }}\right)\right] \mathrm{II}\left[\mathrm{r}_{\text {out } 8}\left(1+\mathrm{g}_{\mathrm{m} 8} \mathrm{r}_{\text {out } 6}\right)\right]
\end{gathered}
$$

The gain of folded cascode op-amp is then

$$
\mathrm{A}=\mathrm{v}_{\text {out }} / \mathrm{v}_{\text {in }}=\mathrm{g}_{\mathrm{m}} \mathrm{R}_{\text {out }} \text {. }
$$

The dominant pole of the op-amp is at $1 / 2 \prod \mathrm{R}_{\text {out }} \mathrm{C}_{\mathrm{L}}$.

Parasitic poles exist at M7/M8 \& M9/M10 combination. These parasitic poles must be larger than the unity gain frequency of the op-amp given by

$$
\mathrm{Fu}=\mathrm{g}_{\mathrm{m}} / 2 \prod \mathrm{C}_{\mathrm{L}}
$$


International Journal of VLSI design \& Communication Systems (VLSICS) Vol.2, No.3, September 2011

For the required unity gain frequency and for a given transistor $g_{m}, C_{L}$ is evaluated using eq. (2).

\subsection{Biasing circuit}

The bias voltages are generated using the circuit shown in fig. (4). The circuit basically has two wide swing current mirrors. M1 and M2 are sized $1 / 4$ of the size of other MOSFETs. If current through resistor, $\mathrm{I}=5 \mathrm{uA}$, then this biasing scheme results in $5 \mathrm{uA}$ to flow in M13 and M14 of figure 3. MOSFETs M1, M2 and M7-M12 will carry 5 uA of drain current. MOSFETs M5/M6 must now carry $7.5 \mathrm{uA}$. This biasing scheme gives maximum swing at the drains of M8/M10. The currents in M5-M6 are 7.5uA. The CMFB circuit must adjust the gate drive of M5 and M6 so that they provide $7.5 \mathrm{uA}$ of current in them.

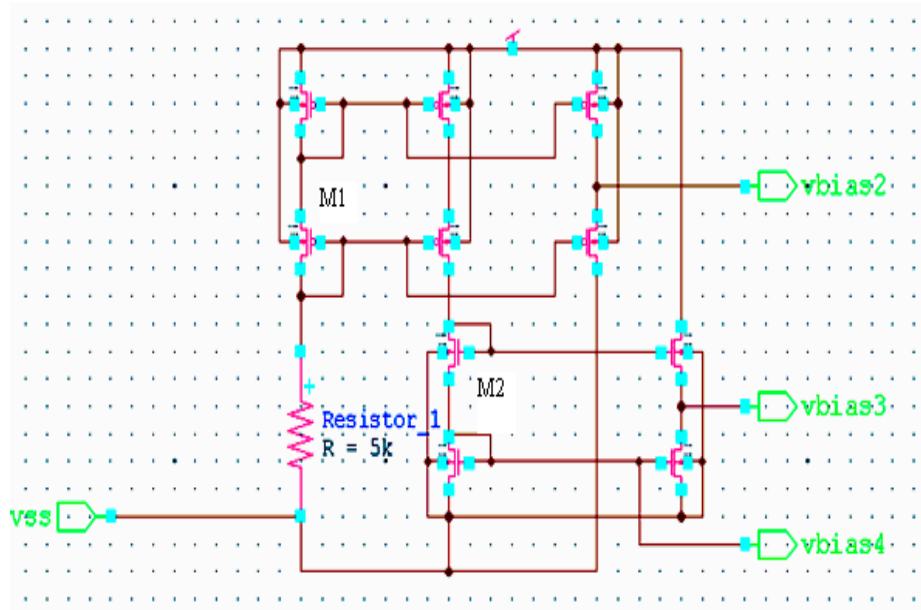

Figure 4. Biasing circuit for the folded cascode op-amp

\subsection{CMFB circuit}

The common mode feedback circuit is shown in figure 5 .

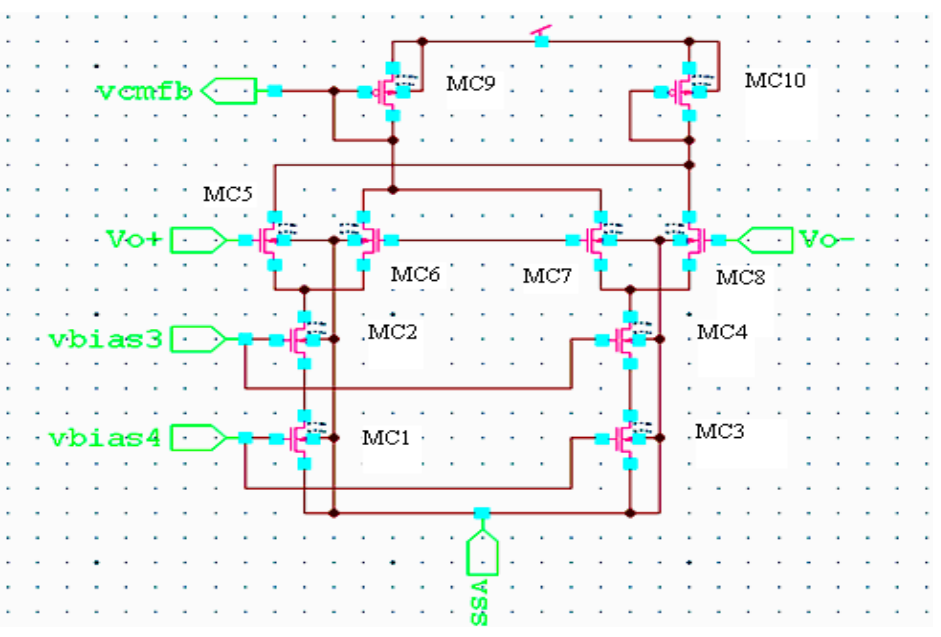

Figure 5. The CMFB circuit 
International Journal of VLSI design \& Communication Systems (VLSICS) Vol.2, No.3, September 2011

Here, the output of folded cascode op-amp, $v_{0+}$ and $v_{\mathrm{o}_{-}}$are the inputs to the CMFB circuit while the output is $\mathrm{V}_{\mathrm{cmfb}}$. This circuit rejects the difference mode signal at its input and amplifies the common mode signal. This is exactly opposite to that of a differential amplifier.

The expression for output is

$$
\mathrm{Vcmfb}=\mathrm{A}\left[\left(\mathrm{v}_{\mathrm{o}+}+\mathrm{v}_{\mathrm{o}^{-}}\right) / 2-\mathrm{Vcm}\right]
$$

In the circuit, if $\mathrm{v}_{\text {out }}$ and $\mathrm{v}_{\text {out- }}$ goes above $\mathrm{Vcm}$, then drain currents of MC6 \& MC7 starts to decrease. This makes $\mathrm{Vcmfb}$ to increase towards $\mathrm{V}_{\mathrm{dd}}$. This increase in $\mathrm{Vcmfb}$ will make the drain currents of M5/M6 of fig (3) to decrease. Since currents in M9 to M12 are constant, it results in a decrease of $\mathrm{v}_{\mathrm{o}_{+}}$and $\mathrm{v}_{\mathrm{o}-\text {. }}$ Similar arguments can be made if vo+ and vo- goes below $\mathrm{Vcm}$.

Figure 6 shows a fully differential $\mathrm{S} / \mathrm{H}$ implementation using an op-amp or an OTA. At $\mathrm{t}=\mathrm{t}_{0}$, the switches $\Phi 1$ and $\Phi 2$ are closed while $\Phi 3$ switches are open. During $\mathrm{t}_{1} \rightarrow \mathrm{t}_{2}$ duration, the input voltage charges the capacitor $\mathrm{C}_{\mathrm{H}}$.

\section{SAMPLE AND HOLD CIRCUIT}

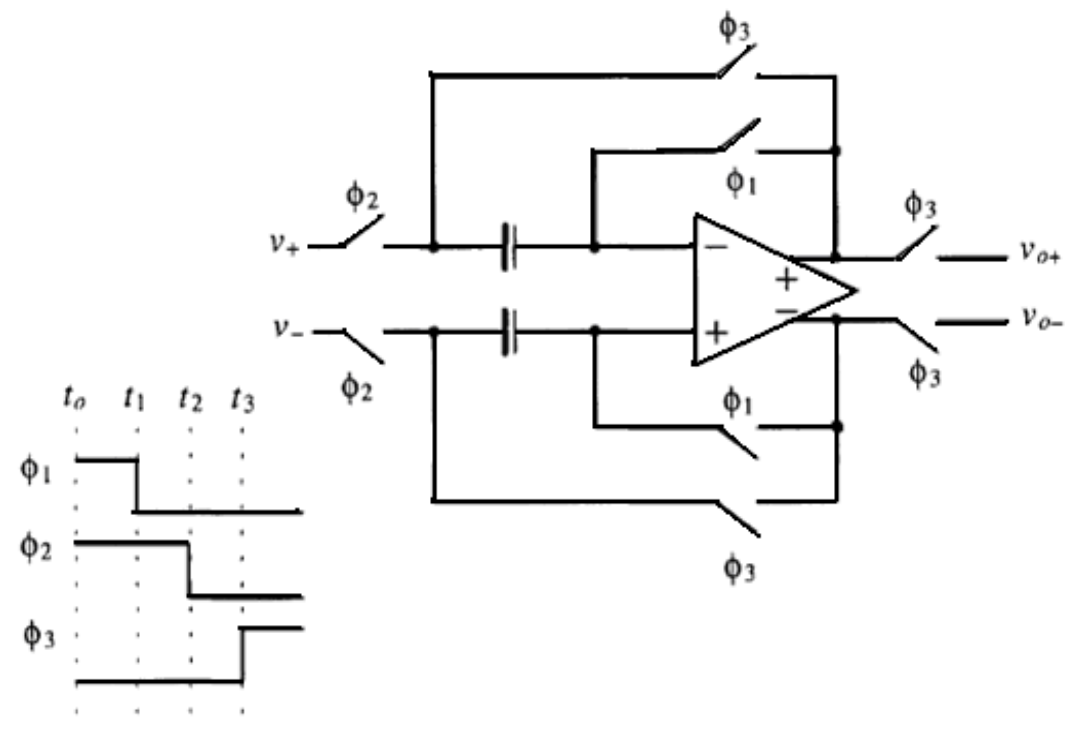

Figure 6. Fully differential sample and hold circuit

At $t 1$, the switch $\Phi 1$ opens and for a very short duration $t_{3} \rightarrow t_{1}$, the op-amp is in open loop [2] [5]. As the top plate of $\mathrm{C}_{\mathrm{H}}$ is at gnd, the charge injection and capacitive feed through resulting from turning OFF of $\Phi 3$ switches becomes independent of input signal. When $\Phi 2$ switch turns OFF, the charge injection will flow into the low impedance vin and not into the right side of $\Phi 2$ as this impedance is large. This leaves the voltage across capacitor unaffected by the charge injection resulting from the turning OFF of the switches. This sequence of turning OFF of the switches is called bottom plate sampling. The fully differential Sample and Hold implementation is shown in fig. (7). We can determine the input/output relationship of sample and hold circuit by evaluating 
International Journal of VLSI design \& Communication Systems (VLSICS) Vol.2, No.3, September 2011 the charge stored on $\mathrm{Ci}$ and $\mathrm{Cf}$. Initially, assuming Vci+ and Vci- are combined and connected to a common voltage $\mathrm{Vcm}$, we have

When $\Phi 1$ and $\Phi 2$ switches are closed and $\Phi 3$ open then the charge on Ci and Cf

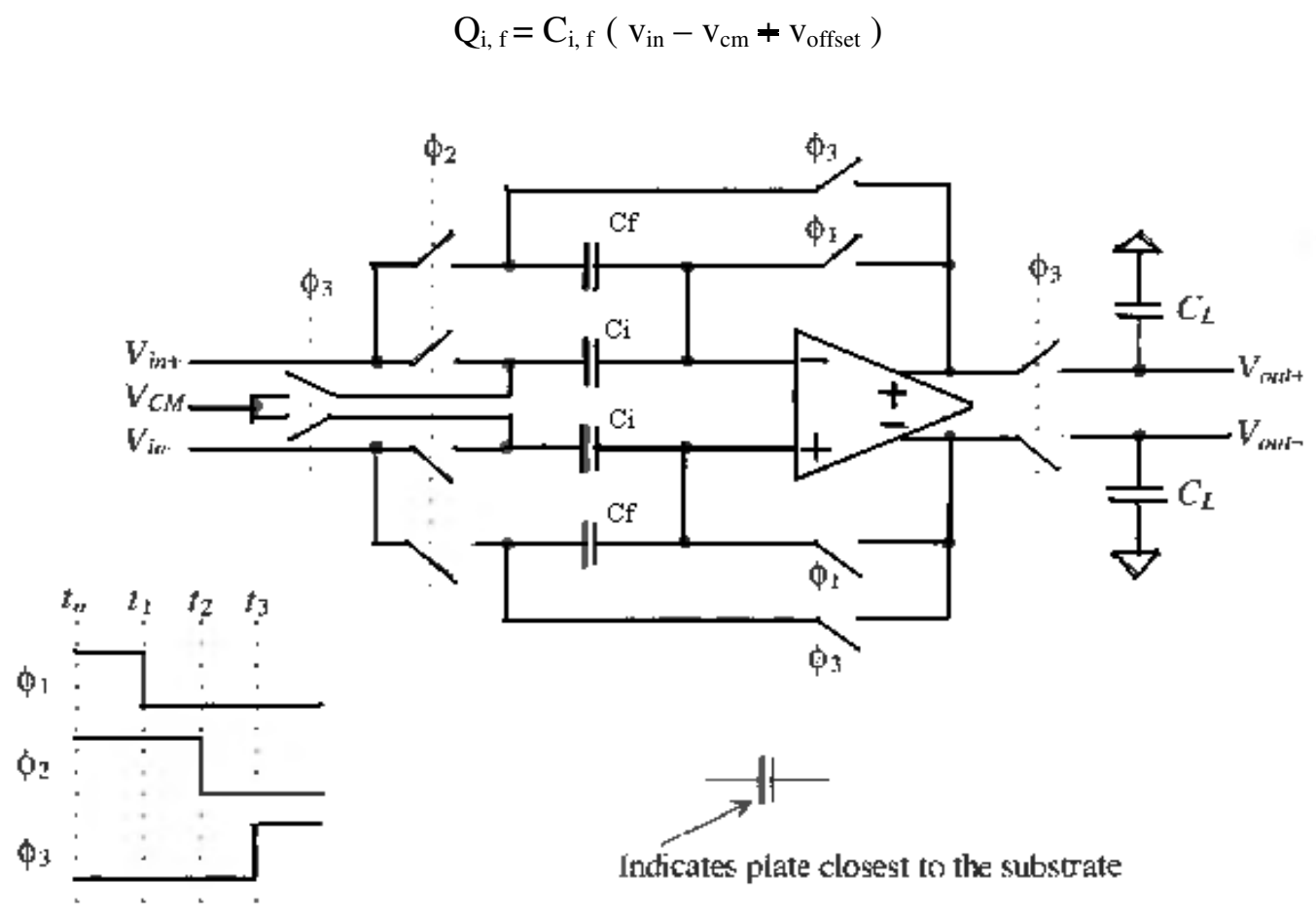

Figure 7. Fully differential S/H circuit implementation

When $\Phi 3$ goes high, the charge on $\mathrm{Ci}$ is

$$
\mathrm{Q}_{\text {in }}=\mathrm{C}_{\mathrm{i}}\left(\mathrm{v}_{\mathrm{cm}}-\mathrm{v}_{\mathrm{cm}}+-\mathrm{v}_{\mathrm{offset}}\right)
$$

The difference between Qin (when $\Phi 1$ is ON ) and Qin ( when $\Phi 3$ is ON is transferred to Cf when $\Phi 3$ goes high. Since the charge must be conserved,

$$
\mathrm{Cf}\left(\text { vout }-\mathrm{vcm} \pm \mathrm{v}_{\text {offset }}\right)=\mathrm{C}_{\mathrm{f}}\left(\mathrm{v}_{\text {in }}-\mathrm{v}_{\mathrm{cm}} \pm \mathrm{v}_{\text {offset }}\right)+\mathrm{C}_{\mathrm{i}}\left(\mathrm{v}_{\text {in }}-\mathrm{v}_{\mathrm{cm}} \pm \mathrm{v}_{\text {offset }}\right)
$$

OR when $\Phi 3$ is on,

$$
+C_{i}\left(v_{c m}-v_{c m} \pm v_{\text {offset }}\right)
$$

$$
\text { Vout }=[1+\mathrm{Ci} / \mathrm{Cf}] \text { vin }-\mathrm{Ci} / \mathrm{Cf} \mathrm{vcm} \ldots .
$$

Notice that the op-amp offset is automatically zeroed out.

Eq. 3 can be used to find the relation of vin and vout for fully differential signals given by

$$
\text { Vout }=(\text { vout }+- \text { vout }-)=(1+\mathrm{Ci} / \mathrm{Cf})(\text { vin }+- \text { vin }-)
$$


International Journal of VLSI design \& Communication Systems (VLSICS) Vol.2, No.3, September 2011

Notice that the common mode voltage subtracts out.

If $\mathrm{v}_{\mathrm{cm}}$ is replaced by $\mathrm{Vci}+$ and Vci- as shown in figure 8 , then

$$
\begin{gathered}
\text { Vout }+=(1+\mathrm{Ci} / \mathrm{Cf}) \text { vin }+-\mathrm{Ci} / \mathrm{Cf} \text { Vci+ and } \\
\text { Vout }=(\text { vout }+- \text { vout }-)=(1+\mathrm{Ci} / \mathrm{Cf})(\text { vin }+- \text { vin }-)-\mathrm{Ci} / \mathrm{Cf}(\text { Vci }+- \text { Vci- })
\end{gathered}
$$

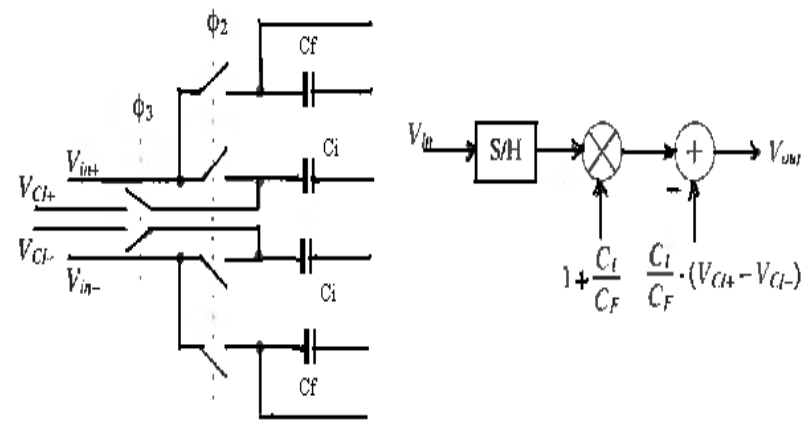

Figure 8. Implementing subtraction in $\mathrm{S} / \mathrm{H}$

\section{1.5 BITS / STAGE}

Pipelined ADCs get their final resolution using a series of cascaded lower resolution stages [3][8][9]. For example, a 12 bit ADC can be a cascade of four 3-bit stages. Many designers are comfortable with 3-bit flash ADCs. However, 1.5 bits / stage is also becoming increasingly popular. For high speed converters there is an advantage of going for minimum stage resolution. It minimises the interstage gain required, which in turn maximises the bandwidth, since gain bandwidth product is a constant for a given technology.

A $1.5 \mathrm{bits} / \mathrm{stage}$ is a 1bit/stage into which some redundancy is added to provide for device tolerances and imperfections. A digital error correction later eliminates this redundancy. The 1.5bits/stage uses two analog comparison levels $\mathrm{Vu} \& \mathrm{VL}$ instead of a single level in a 1 bit/stage. Because of the introduction of gain of two, they must lie between - Vref/2 and + Vref/2. a common choice is $\mathrm{Vu}=+\mathrm{Vref} / 4$ and $\mathrm{VL}=-\mathrm{Vref} / 4$.

The voltage transfer characteristic is shown in fig. 9 (b) which is highly nonlinear.

The input voltage range is divided into three sections. The upper range (U) above $\mathrm{Vu}$, mid range between $\mathrm{Vu}$ and $\mathrm{V}_{\mathrm{L}}$ and low range $(\mathrm{L})$ below $\mathrm{V}_{\mathrm{L}}$ as shown in the table 1 .

Table1.

\begin{tabular}{|l|l|l|l|l|c|}
\hline Vin & Range & B1 & B0 & DAC o/p & Analog residue o/p \\
\hline Vin $>\mathrm{Vu}$ & U & 1 & 0 & + Vref & 2Vin - Vref \\
\hline $\mathrm{V}_{\mathrm{L}}<$ Vin $<\mathrm{Vu}$ & M & 0 & 1 & 0 & 2Vin \\
\hline Vin $<\mathrm{V}_{\mathrm{L}}$ & L & 0 & 0 & - Vref & 2Vin + Vref \\
\hline
\end{tabular}


International Journal of VLSI design \& Communication Systems (VLSICS) Vol.2, No.3, September 2011

The implementation of 1.5bits/stage is shown in fig. 9 ( a ). A resistor string provides voltage division to create reference voltages. All other high accuracy operations such as multiply-by-two are achieved by capacitor ratios. The sample and hold circuit and multiply-by-two amplifier can be combined to form a multiplying DAC ( MDAC ). The cascaded MDAC outputs are passed through latches before fed to the redundancy bit removal circuit as shown in figure11.

\subsection{Redundancy bit removal algorithm}

The probable error sources in data converters include offset voltages in comparators and op-amps, gain error in amplifier, nonlinearity in converter and others. Many of these errors are corrected by this algorithm[8][9].

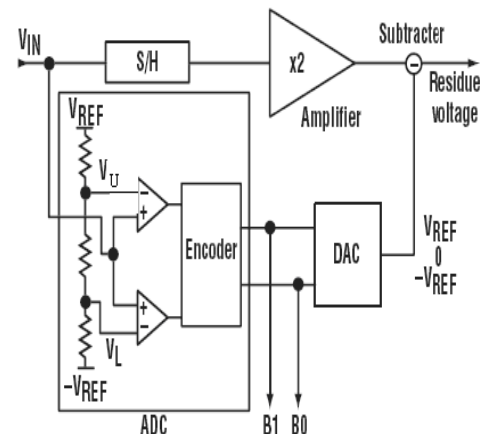

(a)

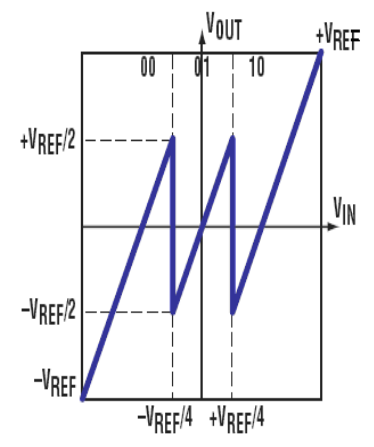

(b)

Figure 9. (a) A 1.5 bit pipelined ADC stage. (b)The stage voltage transfer characteristic.

Each 1.5bit pipelined stage produces a 2 bit code B1B0. Using redundancy bit removal algorithm, this is reduced to final 1 bit per stage code. For a resolution of 3 bits, the input voltage range of $\neq 2 \mathrm{~V}$ is divided into 8 equal slots and the input voltage, the code generation of each stage and corresponding stage residue voltages are shown in table 2 . To generate the final code, the two bit codes generated by each stage are added in a systematic way. For example, as highlighted in table 2 , for $\mathrm{Vin}=1.33 \mathrm{~V}$, the codes generated by successive stages are 10,10 and 00 .

Table 2. Development of error corrected output code

\begin{tabular}{|c|c|l|l|l|l|l|l|l|l|l|l|}
\hline $\begin{array}{c}\text { INPUT } \\
\text { RANGE } \\
(\mathrm{V})\end{array}$ & $\begin{array}{l}\text { DESIGN } \\
\text { OUTPU- } \\
\text { TCODE }\end{array}$ & $\begin{array}{l}\text { VIN } \\
\mathrm{V})\end{array}$ & $\begin{array}{l}\text { RANGE } \\
1\end{array}$ & $\begin{array}{l}\text { CODE } \\
1\end{array}$ & $\begin{array}{l}\text { RES } \\
1\end{array}$ & $\begin{array}{l}\text { RANGE } \\
2\end{array}$ & $\begin{array}{l}\text { CODE } \\
2\end{array}$ & $\begin{array}{l}\text { RES } \\
2\end{array}$ & $\begin{array}{l}\text { RANG- } \\
\text { E3 }\end{array}$ & $\begin{array}{l}\text { CODE } \\
3\end{array}$ & $\begin{array}{l}\text { OUTIVED } \\
\text { OUTP } \\
\text { CODE }\end{array}$ \\
\hline 2.00 & 111 & 1.70 & $\mathrm{H}$ & 10 & 1.40 & $\mathrm{H}$ & 10 & 0.80 & $\mathrm{H}$ & 10 & 111 \\
\hline 1.50 & 110 & 1.33 & $\mathrm{H}$ & 10 & 0.66 & $\mathrm{H}$ & 10 & -0.68 & $\mathrm{~L}$ & 00 & 110 \\
\hline 1.00 & 101 & .79 & $\mathrm{H}$ & 10 & -0.42 & $\mathrm{M}$ & 01 & -0.84 & $\mathrm{~L}$ & 00 & 101 \\
\hline 0.50 & 100 & .19 & $\mathrm{M}$ & 01 & 0.38 & $\mathrm{M}$ & 01 & 0.76 & $\mathrm{H}$ & 10 & 100 \\
\hline 0.00 & 011 & -0.35 & $\mathrm{M}$ & 01 & -0.70 & $\mathrm{~L}$ & 00 & 0.60 & $\mathrm{H}$ & 10 & 011 \\
\hline-0.50 & 010 & -0.68 & $\mathrm{~L}$ & 00 & 0.64 & $\mathrm{H}$ & 10 & -0.72 & $\mathrm{~L}$ & 00 & 010 \\
\hline-1.50 & 001 & -1.21 & $\mathrm{~L}$ & 00 & -0.42 & $\mathrm{M}$ & 01 & -0.84 & $\mathrm{~L}$ & 00 & 001 \\
\hline-2.00 & 000 & -1.82 & $\mathrm{~L}$ & 00 & -1.64 & $\mathrm{~L}$ & 00 & -1.28 & $\mathrm{~L}$ & 00 & 000 \\
\hline
\end{tabular}


International Journal of VLSI design \& Communication Systems (VLSICS) Vol.2, No.3, September 2011 These bits must be added as follows to generate the final 3 bit code.

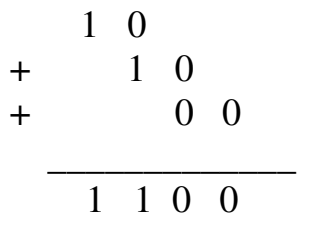

Discard LSB and the final digital code is 110 for the case Vin $=1.33 \mathrm{~V}$. The circuit to implement this algorithm is shown in fig. (10).

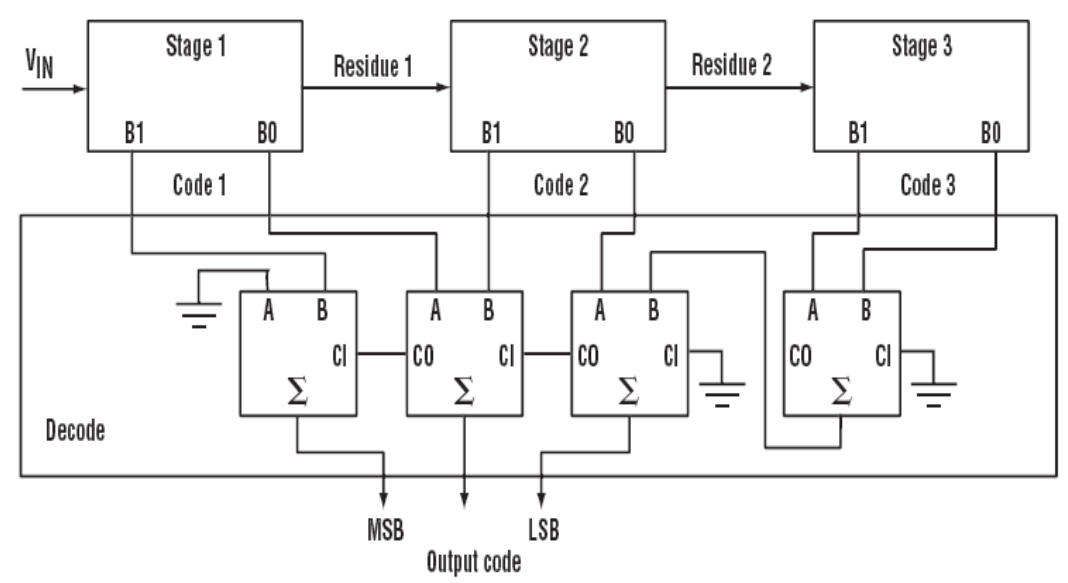

Figure10. Implementation of redundancy bit removal algorithm

\section{RESULTS}

The gain and phase margin curves are as shown in figure 11 where the gain in $75 \mathrm{~dB}$ and the unity gain frequency is $200 \mathrm{MHz}$ at a phase margin of $88^{0}$. The differential outputs of $100 \mathrm{Msps}$ sample and hold circuit with a gain of two are shown in figure 12.

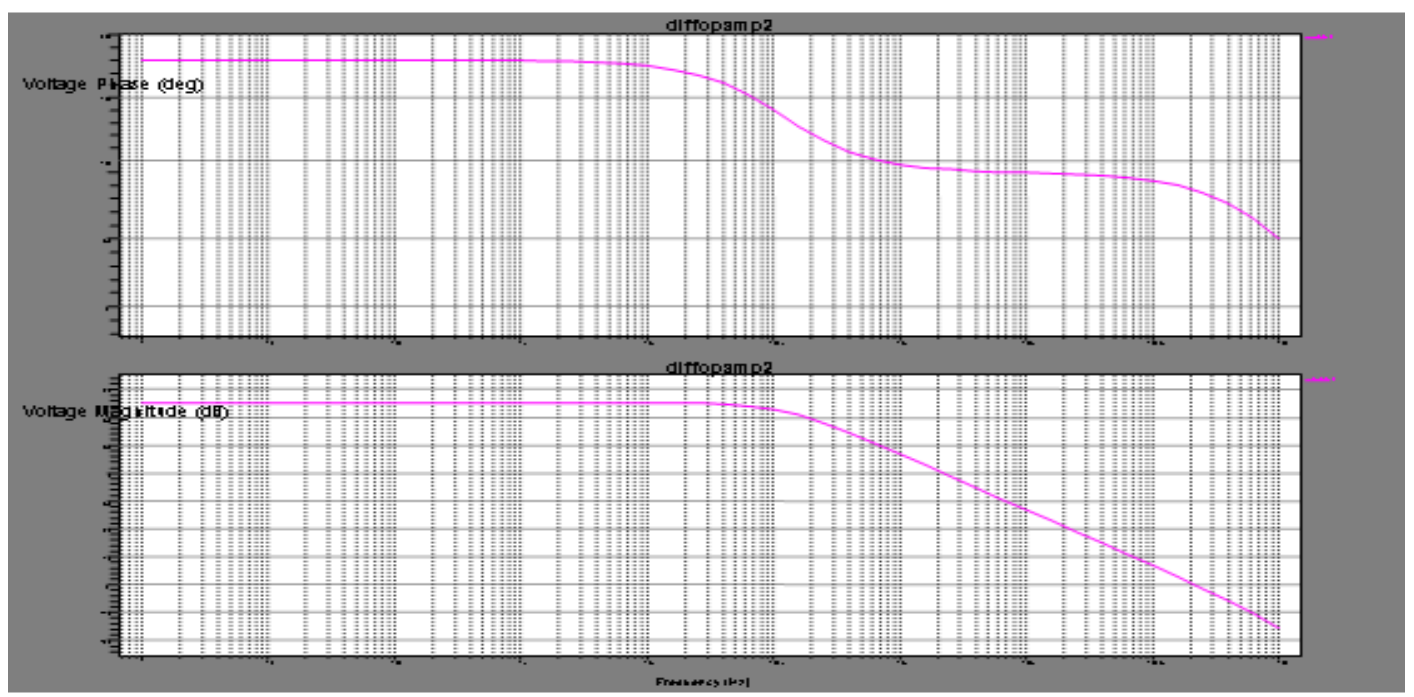

Figure 11. Gain and phase plots of folded cascode op-amp 
International Journal of VLSI design \& Communication Systems (VLSICS) Vol.2, No.3, September 2011

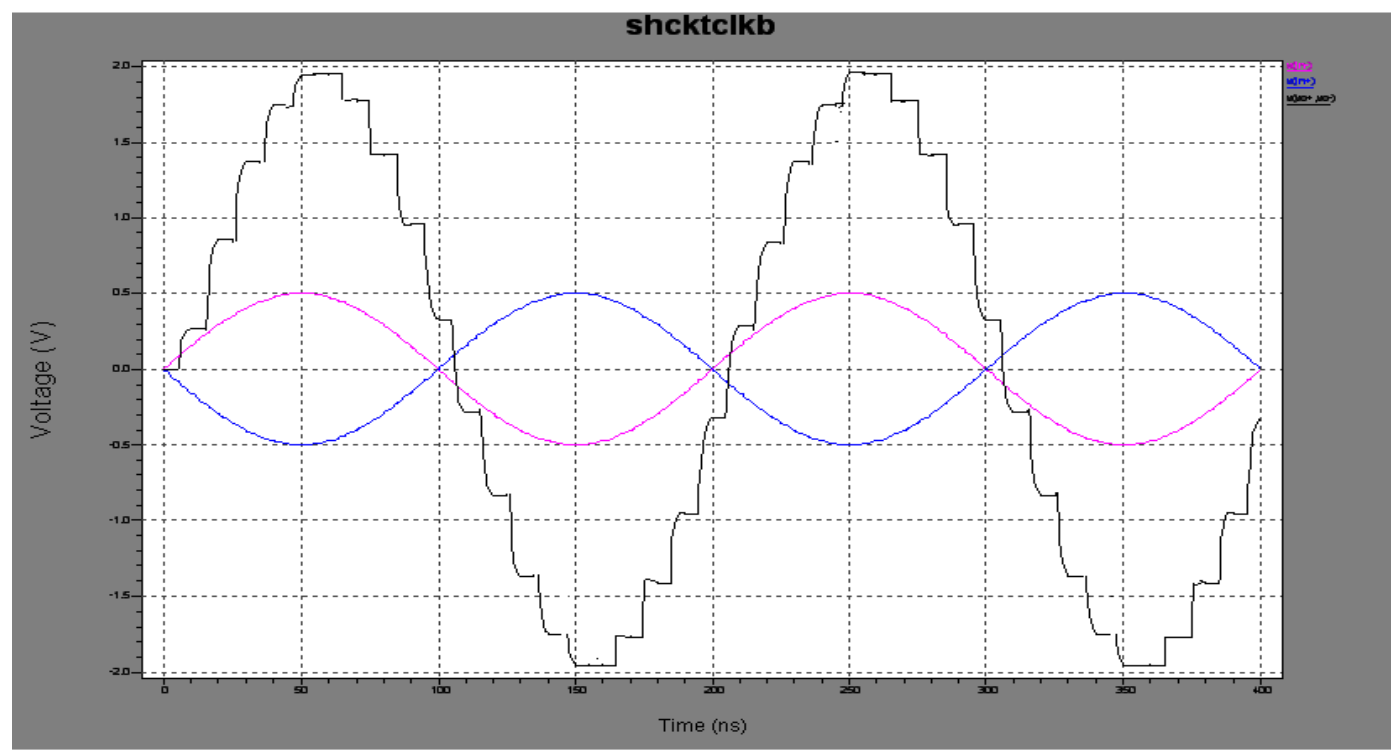

Figure 12. Sample and Hold outputs

The input signals are $0.5 \mathrm{~V}$ differential and the output is seen to be $2 \mathrm{~V}$ i.e. the differential input signals are sampled and given a gain of 2.The INL and DNL errors are observed to be less than $1 / 2$ LSB as in figure 13.
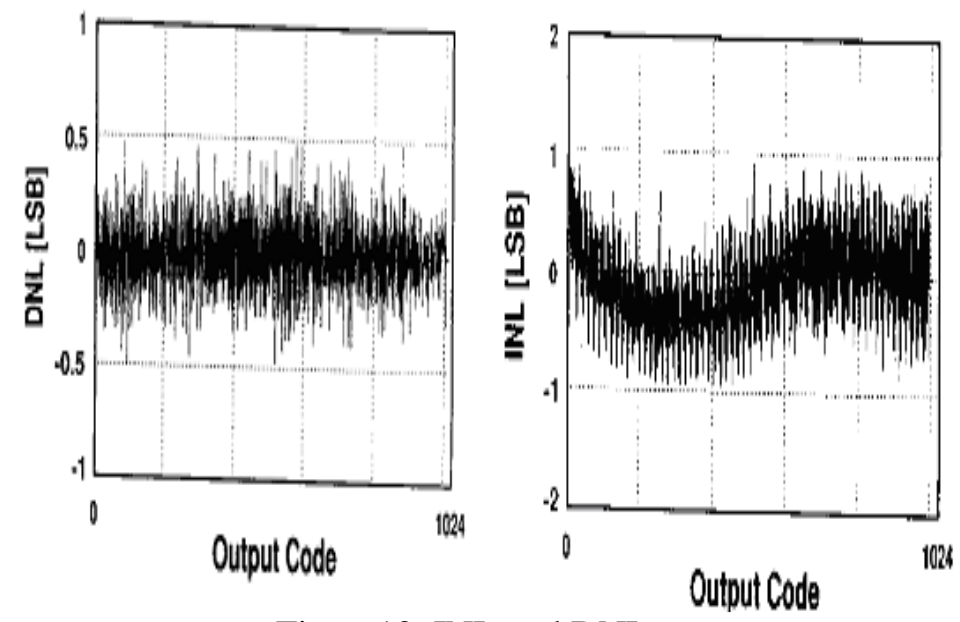

Figure13. INL and DNL error

\section{REFERENCES}

[1] R. Jacob Baker, CMOS mixed signal circuit design, 2nd ed., IEEE press, 2003.

[2] Rudy van de Plassey., CMOS Analog-toDigital and Digital-to-analog Converters, : Springer, 2005.

[3] David A Johns and Ken Martin, Analog integrated circuit design., 2005. 
International Journal of VLSI design \& Communication Systems (VLSICS) Vol.2, No.3, September 2011

[4] Behzad Razavi, Design of Analog CMOS Integrated circuits,TMH 2002.

[5] Jipeng Li and Un-Ku Moon, "A 1.8V 67mW 10bit $100 \mathrm{M} / \mathrm{S}$ Pipelined ADC using time shifted CDS technique,” IEEE J solid state circuits,vol 39 pp. 1468-1476, September 2004.

[6] Thomas Byunghak Cho, Paul R.Gray, "A 10b, 20 Msample/s, $35 \mathrm{~mW}$ Pipeline A/D Converter", IEEE Journal of Solid State Circuits, Vol. 30, No.3, March 1995

[7] Byung-Moo Min, Peter Kim, Frederick W. Bowman, David M. Boisvert, "A 69 mW 10 bit 80 Msps Pipelined CMOS ADC”, IEEE Journal of Solid State Circuits, Vol. 38, No.12, December 2003.

[8] Hung-Chih Liu, Zwei-Mei Lee, Jieh-Tsorng Wu, "a 15b 20MS/s CMOS Pipelined ADC with Digital background Calibration”, ISSCC 2004.

[9] Ming-Huang Liu, Kuo-Chan Huang, Wei-Yang Ou, Tsung-Yi Su, Shen-Iuan Liu, “A Low VoltagePower 13 bit 16 MSPS CMOS Pipelined ADC", IEEE Journal of Solid State Circuits, Vol. 39, No.5, May 2004.

[10] Ratul Kr. Baruah, “ Design of Low power low voltage CMOS opamp”, International Journal of VLSI design \& Communication Systems (VLSICS) vol.1 N0.1 March 2010.

\section{Authors}

Mr.P.Prasad Rao is research Scholar in JNT University, Hyderabad and having more than 19 years of teaching experience. He is presently working as an Associate Professor at SR Engineering College, Warangal, AP. He was Awarded Fellowship in VLSI Design twice in 2006 \& 2007. He has published papers in many national conferences and in international journal. Mr.P.Prasad Rao has post-Graduate Degree in VLSI System Design from National Institute of Technology, Warangal.

Prof. K. Lal Kishore is a Senior Professor in Electronics and Communications Engg., Department of JNTUH University, Hyderabad and is presently the Director, R \& D Cell. He has more than 114 Research Publications to his credit. He has produced Six Ph.Ds and many more Research Scholars are working under his Guidance. He has over 33 years of Experience in Teaching and Research. Prof. K. Lal Kishore has Post-Graduate and Ph.D Degrees from Indian Institute of Science (I.I.Sc) Bangalore. He had held number of administrative positions in the University

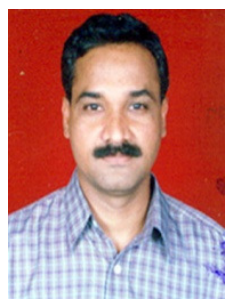
including that of Rector, Registrar, Director, Academic and Planning, Director, School of IT, Principal JNTUH etc.

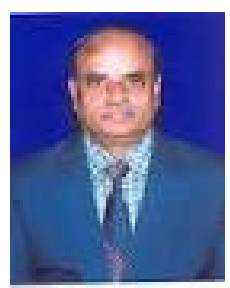

